\title{
First report of a Staphylococcus caprae isolated from middle ear fluid of an infant with recurrent acute otitis media
}

\author{
Elżbieta Mazur ${ }^{1}$, Piotr Żychowski ${ }^{2}$, Marek Juda ${ }^{3}$, Izabela Korona-Głowniak ${ }^{3}$, \\ Grażyna Niedzielska², Anna Malm ${ }^{3}$, Maria Kozioł-Montewka' \\ ${ }^{1}$ Medical Microbiology Department, Medical University of Lublin, Poland \\ ${ }^{2}$ Department of Paediatric Otolaryngology, Phoniatrics and Audiology, Medical University of Lublin, Poland \\ 3 Pharmaceutical Microbiology Department, Medical University of Lublin, Poland
}

Mazur E, Żychowski P, Juda M, Korona-Głowniak I, Niedzielska G, Malm A, Kozioł-Montewka M. First report of a Staphylococcus caprae isolated from middle ear fluid of an infant with recurrent acute otitis media. Ann Agric Environ Med. 2017; 24(3): 357-359. doi: 10.5604/12321966.1233553

\begin{abstract}
Staphylococcus caprae was originally isolated from goat milk. This uncommon coagulase-negative staphylococcus, usually associated with animals, has only infrequently been detected in human clinical specimens. Its association with acute otitis media has not been demonstrated so far. The study reports the first isolation of S. caprae from the middle ear fluid of a 12-month-old infant with recurrent, bilateral acute otitis media. Biochemical traits and susceptibility pattern of the isolated strain are also presented.
\end{abstract}

\section{Key words}

Staphylococcus caprae, recurrent acute otitis media, middle ear fluid, antimicrobial susceptibility, biochemical profile

\section{INTRODUCTION}

Staphylococcus caprae was originally isolated from goat milk by Devriese et al. in 1983 [1]. It is the predominant species among coagulase-negative staphylococci colonizing the skin covering the udders of healthy goats [2]. Since the time of its first isolation, only infrequent cases of human infections caused by $S$. caprae have been reported, among them endocarditis, sepsis, urinary tract infections, bone and joint infections [2-5]. Recently, meningitis following the infection of an intraspinal device and central lineassociated bacteraemia have been described $[6,7]$. One case of mastoiditis and cases of acute otitis externa have also been documented $[8,9]$. Reported human S. caprae infections have been acquired nosocomially following the administration of antibiotics [2,3]. The presented study reports the first isolation of S.caprae from the middle ear fluid (MEF) of a 12-month-old infant with recurrent, bilateral acute otitis media (AOM).

\section{CASE REPORT}

The patient was a 12-month-old male who presented to the paediatric emergency room with rhinorrhea and cough. He was weepy, anxious, agitated, yet afebrile. His parents reported that these symptoms appeared two days before and developed despite symptomatic treatment with paracetamol. In the medical history there were 4-5 AOM episodes during the last five months, diagnosed by general practitioners and treated with antibiotics. The last AOM incident, which occurred about one month before, was treated in the hospital

Address for correspondence: Marek Juda, Pharmaceutical Microbiology Department, Medical University of Lublin, Chodźki 1, 20-093 Lublin, Poland E-mail:marek.juda@umlub.pl

Received: 27 April 2014; accepted: 14 November 2014; first published: February 2017 with intravenous cefotaxime for 5 days. The child did not receive a conjugated pneumococcal vaccine.

On paediatric examination the patient had normal vital signs. Laryngological examination revealed hyperaemia of throat mucosa and nasal concha, as well as abundant sero-mucous secretion in both nasal passages. Otoscopy revealed bilateral AOM. Tympanic membrane of the right ear was congested, matt, entirely bulged, tense; of the left ear: grey-pink, matt, bulged in lower quadrants. Objective hearing examination was performed. Distortion product otoacoustic emission was absent bilaterally. The presence of inflammatory exudate in middle ear was confirmed in impedance audiometry: bilateral type B tympanogram, no reflexes from stapedial muscles. Blood cell count revealed leukocytosis $\left(21,380 / \mathrm{mm}^{3}\right)$ with increased values of both: neutrophils $\left(5,460 / \mathrm{mm}^{3}\right)$ and lymphocytes $\left(14,490 / \mathrm{mm}^{3}\right)$. Other blood parameters were normal.

The boy was admitted to the hospital and classified for emergency operation under general anaesthesia. Antisepsis of the ear canal before the procedure was performed by installing an Octenisept (Schülke\&Mayr) for 1 minute. After its removal by suction, myringotomy was carried out, during which a dense, mucopurulent discharge was found bilaterally draining under pressure from middle ear. An MEF sample was collected from each ear with the use of a sterile suction needle inserted into middle ear cavity through an incision in the tympanic membrane. Both samples were placed in sterile Eppendorf tubes, maintained at air temperature, and transported immediately to the laboratory. The remaining amount of exudate was aspirated. Next, ventilation drainage of middle ear was placed bilaterally. Oral amoxicillin (90 mg/kg/day) and anti-inflammatory treatment was started.

Twenty-four hours after the operation the infant was discharged home in a good general and local state, with the recommendation to present at a follow-up visit after 2-3 days in order to assess the local state and, if necessary, 
to correct antibiotic therapy depending on the results of microbiological examination. The patient presented after 2 weeks with no signs of upper respiratory tract infection. According to the parents' report, treatment with amoxicillin was carried out for 8 days. Both tympanic membranes were grey, with vestigial light reflex. Ventilation drainage was permeable, without any pathological discharge. Behavioural hearing tests were performed, no deviation from the normal state was noted.

Bacteriology findings. In the right and left MEF samples, respectively, pure cultures of $S$. pneumoniae and $S$. caprae were identified with the use of routine microbiological methods. Antimicrobial susceptibility results were interpreted according to the European Committee on Antimicrobial Susceptibility Testing recommendations (EUCAST 2012, version 2.0) [10]. S. pneumoniae strain was specified as serotype $19 \mathrm{~F}$ by means of quellung reaction using antisera provided by Statens Serum Institute (Copenhagen, Denmark). Resistance to oxacillin, erythromycin, tetracycline, clindamycin, and trimethoprim/sulfamethoxazole was detected with the use of a disc diffusion method. The strain was classified as penicillin non-susceptible based on benzylpenicillin minimal inhibitory concentration (MIC $=0.75 \mathrm{mg} / \mathrm{L}$ ) evaluated by using the E-test (AB Biodisk, Sweden). Identification of $S$. caprae and its drug susceptibility were performed by Vitek 2 Compact (bioMérieux) using GP and AST-P580 cards (Bionumber: 010002002661221; 99\% probability). Tables 1 and 2, respectively, show the biochemical traits and antimicrobial susceptibility of the isolated strain.

Table 1. Biochemical activity of Staphylococcus caprae strain isolated from middle ear fluid

\begin{tabular}{lc|lc}
\hline Test or characteristic & Result & Test or characteristic & Result \\
\hline Haemolysis of sheep blood & + & Enzymatic activity of: & \\
\hline Production of acid from: & & Phosphatidylinositol & - \\
D-amygdalin & - & phospholipase C & + \\
D-xylose & - & Arginine dihydrolase & - \\
Cyclodextrin & - & Beta-galactosidase & - \\
D-sorbitol & - & Alpha-glucosidase & - \\
D-galactose & - & Ala-Phe-Pro aryloamidase & - \\
D-ribose & - & L-aspartate aryloamidase & - \\
L-lactate & + & Beta-galactopyranosidase & - \\
Lactose & - & Alpha-mannosidase & - \\
N-acetyl-D-glucosamine & - & Phosphatase & - \\
D-maltose & + & Leucine aryloamidase & - \\
D-mannitol & + & L-proline aryloamidase & - \\
D-mannose & + & Beta-glucoronidase & - \\
Methyl-beta-D-glucopyranoside & - & Alpha-galactosidase & + \\
Pullulan & - & L-pyrrolidonyl-aryloamidase & - \\
D-raffinose & - & Alanine aryloamidase & - \\
Salicin & - & Tyrosine aryloamidase & - \\
Saccharose & - & Urease & - \\
D-trehalose & + & Arginine dihydrolase & \\
\hline Resistance to: & & Growth in 6.5\% NaCl & + \\
Polymyxin B & - & & \\
Bacitracin & + & & \\
Novobiocin & - & & \\
\hline & & & \\
\hline
\end{tabular}

- negative reaction; + positive reaction
Table 2. Antimicrobial susceptibility of Staphylococcus caprae strain isolated from middle ear fluid

\begin{tabular}{lcc}
\hline Antimicrobial & MIC $(\mathrm{mg} / \mathrm{L})$ & Interpretation \\
\hline Benzylpenicillin & $\geq 0.5$ & $\mathrm{R}$ \\
\hline Erythromycin & $\leq 0.25$ & $\mathrm{~S}$ \\
\hline Clindamycin & $\leq 0.25$ & $\mathrm{~S}$ \\
\hline Tetracycline & $\leq 1$ & $\mathrm{~S}$ \\
\hline Fosfomycin & $\geq 128$ & $\mathrm{R}$ \\
\hline Fusidic acid & $\leq 0.5$ & $\mathrm{~S}$ \\
\hline Trimetoprim/sulfamethoxazole & $\leq 10$ & $\mathrm{~S}$ \\
\hline Gentamycin & $\leq 0.5$ & $\mathrm{~S}$ \\
\hline Tobramycin & $\leq 1$ & $\mathrm{~S}$ \\
\hline Levofloxacin & 0.25 & $\mathrm{~S}$ \\
\hline Moxifloxacin & $\leq 0.25$ & $\mathrm{~S}$ \\
\hline Vancomycin & 1 & $\mathrm{~S}$ \\
\hline Teicoplanin & $\leq 0.5$ & $\mathrm{~S}$ \\
\hline Linezolid & 1 & $\mathrm{~S}$ \\
\hline Tigecycline & $\leq 0.12$ & $\mathrm{~S}$ \\
\hline Cefoxitin screen & $\mathrm{NEG}$ & $\mathrm{MSCNS}$ \\
\hline -resistantS suscer & \\
\hline
\end{tabular}

$\mathrm{R}$ - resistant; S - susceptible; MSCNS - methicillin-sensitive coagulase-negative staphylococci

\section{DISCUSSION AND CONCLUSIONS}

Streptococcus pneumoniae remains one of the most important bacterial pathogens in non-complicated as well as recurrent or persistent AOM. In recalcitrant AOM, pneumococcal strains with higher penicillin MICs or multidrug-resistant proved to be more frequently isolated $[11,12]$. Indeed, the S. pneumoniae 19F strain was isolated in the MEF obtained from the right ear of the presented patient; the isolate turned out to be penicillin non-susceptible and multidrug-resistant. However, in the left MEF sample, the copious growth of $S$. caprae was detected in pure culture. Simultaneous isolation of 2 different bacteria from each MEF sample proved to be an extremely rare event in bilateral AOM $[11,12]$. Nonetheless, in one out of 26 recalcitrant bilateral AOM episodes reported by Pumarola et al. [11], pure cultures of prominent otopathogens, namely S. pneumoniae and Haemophilus influenzae, were yielded from each MEF sample, showing that infection in both ears is not necessarily caused by the same bacterial species. Hence, the isolation of S. caprae in pure culture, together with otoscopically-confirmed AOM signs, strongly suggests its etiological role in the left middle ear inflammatory process in the presented patient. Although $S$. caprae association with AOM has not been documented so far, its role as a pathogen was demonstrated and pathogenicity determinants were detected, including the ica operon involved in biofilm formation and a gene encoding a fibronectin-binding autolysin [13]. Interestingly, icaoperon and surface proteins binding fibronectin have been detected in all human and goat $S$. caprae isolates tested [2, 13]. Biofilm and slime production in vitro by some human and goat $S$. caprae strains, contributing to their virulence and protection against antibiotic access, have also been documented [2]. Human infections with $S$. caprae proved to be associated with previous administration of antibiotics and hospitalization $[2,14]$, which revealed their opportunistic 
nature. Of note, both risk factors were present in the current case. The isolated strain proved to be resistant only to penicillin and fosfomycin, the latter feature being considered a helpful presumptive test for the identification of $S$. caprae $[2,14]$. The susceptibility pattern of the isolate in the current case turned out to be similar to those reported by Vandenesch et al. [14], Roland et al. [9] and Kini et al. [4]. However, meticillin-resistant $S$. caprae strains have also been reported $[7,15]$. The isolate in the current case proved to produce acid from mannitol, similar to $100 \%, 87 \%$ and over $90 \%$ of the human strains of $S$. caprae reported by Vandenesh et al. [14], Shuttleworth et al. [8] and Kawamura et al. [3], respectively. In contrast to most human $S$. capraestrains described by Vandenesh et al. [14], and similarly to 53\% of those reported by Kawamura et al. [3], the isolate in the current case showed beta-hemolysis on sheep blood agar. Urease activity has been detected in $100 \%, 93 \%$ and over $90 \%$ of the human S. caprae strains reported by Vandenesh et al. [14], Shuttleworth et al. [8], and Kawamura et al. [3], respectively. The strain isolated from the patient in the current case proved to be urease negative.

The underlying microbiology of AOM is known to change over time in response to various selective pressures, including antibiotic consumption $[11,12]$. The presented report shows that in patients with recurrent AOM, exposed to several courses of antibiotics and hospitalized, antibiotic pressure not only selects for resistant strains among recognized, but also allows rare opportunistic bacteria, such as $S$. caprae, to invade the middle ear cavity. Moreover, this account underscores the necessity to perform microbiological examination of MEF samples obtained from both ears in cases of refractory bilateral acute otitis media, since inflammatory process in each ear may be caused by different bacterial species.

\section{REFERENCES}

1. Devriese LA, Poutrel B, Killper-Bälz R, Schleifer KH. Staphylococcus gallinarum and Staphylococcus caprae, two new species from animals. Int J Syst Bacteriol.1983; 33: 480-486.
2. Allignet J, Galdbart JO, Morvan A, Dyke KG, Vaudaux P, Aubert S et al. Tracking adhesion factors in Staphylococcus caprae strains responsible for human bone infections following implantation of orthopaedic material. Microbiology 1999; 145 (Pt 8): 2033-2042.

3. Kawamura Y, Hou XG, Sultana F, Hirose K, Miyake M, Shu SE et al. Distribution of Staphylococcus species among human clinical specimens and emended description of Staphylococcus caprae. J Clin Microbiol. 1998; 36: 2038-2042.

4. Kini GD, Parris AR, Tang JS. A rare presentation of sepsis from Staphylococcus caprae. Open Microbiol J. 2009; 3: 67-68.

5. Darrieutort-Laffite C, André V, Leautez S, Tanguy G, Cormier G. Staphylococcus caprae arthritis. Med Mal Infect. 2013; 43(3): 131-132.

6. Benedetti P, Pellizzer G, Furlan F, Nicolin R, Rassu M, Sefton A.Staphylococcus caprae meningitis following intraspinal device infection. J Med Microbiol. 2008; 57(Pt 7): 904-906.

7. Kato J, Mori T, Sugita K, Murata M, Ono Y, Yamane A et al. Central line-associated bacteremia caused by drug-resistant Staphylococcus caprae after chemotherapy for acute myelogenous leukemia. Int J Hematol. 2010; 91: 912-913.

8. Shuttleworth R, Behme RJ, McNabb A, Colby WD. Human isolates of Staphylococcus caprae: association with bone and joint infections. J Clin Microbiol. 1997; 35: 2537-2541.

9. Roland PS, Stroman DW. Microbiology of acute otitis externa. Laryngoscope 2002; 112 (7 Pt 1): 1166-1177.

10. European Committee on Antimicrobial Susceptibility Testing. Breakpoints tables for interpretation of MICs and zone diameters. Version 2.0, valid from 2012.01.01. http://www.eucast.org/fileadmin/ src/media/PDFs/EUCAST_files/Breakpoint_tables/Breakpoint_ table_v_2.0_120221.pdf (access: 2014.04.12)

11. Pumarola F, Marès J, Losada I, Minguella I, Moraga F, Tarragó D et al. Microbiology of bacteria causing recurrent acute otitis media (AOM) and AOM treatment failure in young children in Spain: Shifting pathogens in the post-pneumococcal conjugate vaccination era. Int J Pediatr Otorhinolaryngol. 2013; 77(8): 1231-1236.

12. Dupont D, Mahjoub-Messai F, François M, Doit C, Mariani-Kurkdjian $\mathrm{P}$, Bidet $\mathrm{P}$ et al. Evolving microbiology of complicated acute otitis media before and after introduction of the pneumococcal conjugate vaccine in France. Diagn Microbiol Infect Dis. 2010; 68(1): 89-92.

13. Allignet J, Aubert S, Dyke KG, El Solh N. Staphylococcus caprae strains carry determinants known to be involved in pathogenicity: a gene encoding an autolysin-binding fibronectin and the ica operon involved in biofilm formation. Infect Immun. 2001; 69: 712-718.

14. Vandenesch F, Eykyn SJ, Bes M, Meugnier H, Fleurette J, Etienne J. Identification and ribotypes of Staphylococcus caprae isolates isolated as human pathogens and from goat milk. J Clin Microbiol. 1995; 33: 888-892.

15. Ross TL, Fuss EP, Harrington SM, Cai M, Perl TM, Merz WG. Methicillin-resistant Staphylococcus caprae in a neonatal intensive care unit. J Clin Microbiol. 2005; 43: 363-367. 